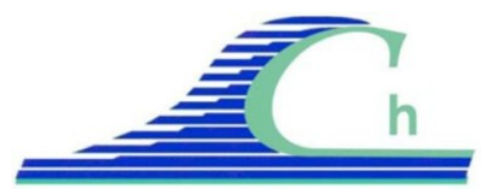

XII ${ }^{\text {ìmes }}$ Journées Nationales Génie Côtier - Génie Civil

Cherbourg, 12-14 juin 2012

DOI:10.5150/jngcgc.2012.001-B C Editions Paralia CFL

disponible en ligne - http://www.paralia.fr - available online

\title{
Retour d'expérience sur l'utilisation des probabilités conjointes pour la caractérisation de niveaux marins à la côte
}

\author{
Thomas BULTEAU ${ }^{1}$, Etienne DELVALLEE ${ }^{2}$, \\ Jérôme THIEBOT ${ }^{3}$, Rodrigo PEDREROS ${ }^{1}$
}

\section{BRGM, Service Risques Naturels/Unité Risques Côtiers,} 3 avenue Claude Guillemin, BP36009 - 45060 Orléans Cedex 2, France. t.bulteau@brgm.fr;r.pedreros@brgm.fr

2. Anciennement BRGM. Désormais à Océanide, Port de Brégaillon, Bâtiment FIRST, BP 63, 83502 La Seyne-sur-Mer, France. edelvallee@oceanide.net

3. ESIX Normandie/LUSAC (Université de Caen), Site universitaire de Cherbourg, BP 78, 50130 Octeville, France.

jerome.thiebot@unicaen.fr

\section{Résumé :}

Le calcul des périodes de retour de niveaux marins à la côte est primordial pour la caractérisation des submersions marines et la gestion des risques côtiers. Or, les submersions marines sont des événements complexes qui dépendent de plusieurs phénomènes partiellement liés : la marée, les surcotes atmosphériques et les vagues. L'étude d'une seule variable n'est donc pas suffisante pour déterminer la période de retour d'un niveau marin à la côte. L'analyse en probabilités conjointes permet de prendre en compte la dépendance entre les variables et ainsi de mieux caractériser les conditions hydrodynamiques d'un site donné. Les résultats de l'analyse statistique peuvent ensuite servir de base pour améliorer les calculs de période de retour de niveaux marins à la côte.

Le présent article est un retour d'expérience sur l'analyse en probabilités conjointes de séries de vagues et de niveaux d'eau au large de Saint-Jean-de-Luz, sur la côte basque française. La méthode mise en œuvre est celle du code Join-Sea, développé par HR Wallingford et l'université de Lancaster. Les résultats de l'analyse statistique sont détaillés, puis deux approches permettant d'estimer la période de retour d'un niveau marin à la côte sont présentées et comparées.

\section{Mots-clés :}

Probabilités conjointes - Niveau marin à la côte - Statistiques des extrêmes - Période de retour - Join-Sea - Saint-Jean-de-Luz 


\section{Introduction}

Les submersions marines sont la conséquence de phénomènes combinés agissant sur les niveaux marins : la marée, les surcotes atmosphériques, les vagues (à travers le setup et le runup notamment). Il arrive ainsi que de violentes submersions ou d'importants dommages aux ouvrages de défense côtière se produisent lors de l'occurrence simultanée de fortes hauteurs de vague et de hauts niveaux d'eau (comprenant la composante marégraphique et les surcotes atmosphériques) sans pour autant que ces deux variables prises individuellement soient exceptionnelles. L'analyse statistique d'une seule variable, telle que le niveau d'eau, n'est donc pas suffisante pour caractériser un niveau marin à la côte. Afin d'améliorer la détermination des périodes de retour de niveaux marins et ainsi permettre une meilleure gestion des risques côtiers, il est nécessaire de prendre en compte la dépendance entre les variables.

La méthode du Department for Environment, Food and Rural Affairs au Royaume-Uni (DEFRA, 2005), dite méthode simplifiée, consiste à estimer les probabilités conjointes de $\mathrm{H}_{\mathrm{s}}$ (hauteur significative des vagues) et SWL (pour Still Water Level, niveau d'eau) à partir des probabilités marginales des deux variables (i.e. des probabilités d'occurrence propres à $\mathrm{H}_{\mathrm{s}}$ d'une part et à SWL d'autre part; les variables sont considérées individuellement). En fonction d'un niveau de dépendance déterminé a priori et d'une période de retour conjointe donnée, une table de valeurs pré-calculées fournit un ensemble de combinaisons possibles de périodes de retour marginales. On peut ainsi déterminer un ensemble de couples de valeurs de $\mathrm{H}_{\mathrm{s}}$, SWL qui ont la période de retour conjointe souhaitée (e.g. $\mathrm{H}_{\mathrm{s}} 10$ ans et SWL 2 ans; $\mathrm{H}_{\mathrm{s}} 1$ an et SWL 20 ans, ...). Cette méthode simplifiée, imprécise et peu flexible, n'est pas applicable à tous les sites. De plus, la détermination a priori du niveau de dépendance peut s'avérer hasardeuse. Une autre méthode plus rigoureuse consiste à entreprendre l'analyse en probabilités conjointes des trois variables partiellement liées $\mathrm{H}_{\mathrm{s}}, \mathrm{T}_{\mathrm{p}}$ (période pic des vagues) et SWL grâce à l'ajustement de modèles statistiques de dépendance. Cette méthode est déjà appliquée depuis 2000 au Royaume-Uni par HR Wallingford (HR WALLINGFORD \& LANCASTER UNIVERSITY, 2000).

Nous appliquons la méthode sur le site de Saint-Jean-de-Luz, sur la côte basque française, à l'aide du code Join-Sea (HAWKES et al., 2002) et à partir de données issues de modèles numériques. Les données utilisées pour l'analyse statistique sont acquises au large (hors déferlement des vagues). Or, pour les submersions marines, il est plutôt utile de disposer d'informations sur les niveaux marins totaux à la côte, c'està-dire des niveaux qui intègrent la marée, la surcote atmosphérique mais également l'effet des vagues (setup et runup). Dans cet article, nous proposons deux méthodes permettant de transférer à la côte, l'information statistique disponible sur les forçages au large, et ainsi de calculer la période de retour de niveaux marins à la côte. 


\section{XII ${ }^{\text {èmes }}$ Journées Nationales Génie Côtier - Génie Civil \\ Cherbourg, 12-14 juin 2012}

\section{Méthode}

\subsection{La méthode Join-Sea}

Join-Sea est un code d'analyse en probabilités conjointes développé par HR Wallingford et l'université de Lancaster à la fin des années 90. Il permet de déterminer la probabilité que les deux variables $\left(\mathrm{H}_{\mathrm{s}}, \mathrm{SWL}\right)$ dépassent conjointement des valeurs données. Join-Sea calcule également la densité de probabilité conjointe des trois variables $\left(\mathrm{H}_{\mathrm{s}}, \mathrm{SWL}, \mathrm{T}_{\mathrm{p}}\right)$ ce qui est particulièrement utile pour calculer des variables de structure. Une variable de structure est une fonction dépendante des trois variables de conditions de mer $\left(\mathrm{H}_{\mathrm{s}}, \mathrm{SWL}, \mathrm{T}_{\mathrm{p}}\right)$. Pour les submersions, le niveau marin total à la côte (Ntot) est une variable de structure pouvant être estimée par :

$N$ tot $=S W L+\operatorname{setup}\left(H_{s}, T_{p}\right)+\operatorname{runup}\left(H_{s}, T_{p}\right)$

La méthode se décline en 5 étapes: (i) Préparation du jeu de données : il s'agit de constituer un échantillon avec un grand nombre de triplets $\left(\mathrm{H}_{s}, \mathrm{SWL}, \mathrm{T}_{\mathrm{p}}\right)$ indépendants. Les triplets correspondent à des enregistrements (observations ou sorties de modèles) des trois variables à un point situé au large, avant le déferlement des vagues. (ii) Ajustement des distributions de probabilité marginales pour $\mathrm{H}_{\mathrm{s}}$ et SWL (loi Generalized Pareto Distribution ou GPD). (iii) Ajustement d'un modèle de dépendance entre $\mathrm{H}_{\mathrm{s}}$ et SWL. Ajustement d'un modèle statistique pour la distribution des cambrures de vague (variable directement reliée à $T_{p}$ et plus robuste pour des calculs statistiques (HAWKES et al., 2002)) en fonction de $\mathrm{H}_{\mathrm{s}}$. (iv) Simulation par la méthode de Monte Carlo d'un très grand nombre de triplets fictifs $\left(\mathrm{H}_{\mathrm{s}}, \mathrm{SWL}, \mathrm{T}_{\mathrm{p}}\right)$ possédant les mêmes caractéristiques statistiques que les données d'origine. (v) Analyse des extrêmes qui comporte deux aspects. Premièrement, Join-Sea peut fournir des points d'iso-période de retour de dépassement conjoint dans l'espace $\left(\mathrm{H}_{\mathrm{s}}, \mathrm{SWL}\right)$. Pour chaque point $(x, y)$ d'un de ces contours d'iso-période de retour, la probabilité de l'évènement $\left\{S W L>x, H_{s}>y\right\}$ est telle que cet évènement se produit en moyenne une fois pendant la période de retour. On a la relation :

$$
P R=1 /\left[N\left(\operatorname{Pr}\left(S W L>x, H_{s}>y\right)\right)\right]
$$

Avec $P R$, la période de retour associée à la probabilité de dépassement conjoint et $N$, le nombre d'observations par an. La variable $T_{p}$ n'intervient pas dans cette analyse. Le deuxième aspect de l'analyse des extrêmes de Join-Sea est la possibilité d'évaluer directement les valeurs extrêmes de variables de structure telles que Ntot, grâce au très grand nombre de triplets simulés. La densité de probabilité conjointe des trois variables $\mathrm{H}_{\mathrm{s}}$, SWL et $\mathrm{T}_{\mathrm{p}}$ est ainsi mise à profit.

\subsection{Calcul de période de retour de niveau marin à la côte}

Une caractéristique essentielle d'une submersion marine est le niveau marin maximal atteint à la côte (e.g. Ntot) pendant l'épisode de tempête. On souhaite donc calculer la 
période de retour de ce niveau marin pour mieux caractériser l'aléa. Or, l'analyse des extrêmes de Join-Sea fournit des informations sur l'occurrence simultanée des forçages au large. Il faut donc trouver une méthode permettant de transférer à la côte, l'information que l'on possède au large. Nous proposons deux approches pour y parvenir.

La première approche consiste à propager des scénarii de forçages au large vers la côte à l'aide d'un modèle numérique afin d'obtenir le niveau marin résultant. Pour cela, on discrétise les courbes d'iso-période de retour de dépassement conjoint calculées par Join-Sea et on associe à chacun de ces points une direction de vague et une période (voir §3.2). Chaque "évènement" $\left(H_{s}, T_{p}, D_{p}, S W L\right)$ représente un scénario de période de retour donnée PR, au large, de dépassement conjoint pour $H_{s}$ et SWL. Le niveau marin maximal atteint à la côte après propagation de tous les scénarii, fournit le niveau marin extrême de période de retour assimilée PR.

La seconde approche consiste à utiliser la densité de probabilité conjointe des variables $\left(\mathrm{H}_{\mathrm{s}}, \mathrm{SWL}, \mathrm{T}_{\mathrm{p}}\right)$ calculée par Join-Sea, pour évaluer directement les extrêmes de la variable de structure Ntot à partir des forçages au large simulés par la méthode de Monte Carlo. Cette approche est basée sur l'utilisation de formules simplifiées pour estimer l'effet des vagues (setup et runup). Citons par exemple les formules empiriques de STOCKDON et al. (2006).

Pour simplifier la démarche, nous nous sommes intéressés uniquement au niveau marin statique, c'est-à-dire sans le runup (voir Eq(1)). Le modèle numérique utilisé dans la première approche est SWAN (modèle développé par l'université de Delft).

\subsection{Présentation du site d'étude et des données utilisées}

Le site choisi pour cette étude est la baie de Saint-Jean-de-Luz, situé au fond du Golfe de Gascogne sur la côte basque. Les données utilisées pour les vagues sont issues de la base de données BoBWA élaborée par Elodie Charles au cours de sa thèse (CHARLES et al., sous presse). Il s'agit d'une ré-analyse effectuée à l'aide du modèle global WaveWatchIII (TOLMAN, 2009) et du modèle SWAN, forcés par les vents issus de la ré-analyse ERA-40. En sortie, nous disposons des triplets de vagues $\left(\mathrm{H}_{\mathrm{s}}, \mathrm{T}_{\mathrm{p}}, \mathrm{D}_{\mathrm{p}}\right)$ sur une période de 45 ans (de 1958 à 2002, toutes les 3h), tous les $2 \mathrm{~km}$ le long de la côte Aquitaine. Le modèle a par ailleurs été validé au niveau de plusieurs bouées MétéoFrance et CANDHIS, dont les bouées Cap-Ferret, Biscarosse et Bayonne.

Pour les séries temporelles de niveaux d'eau, nous effectuons une simulation sur 31 ans (de 1979 à 2009) à l'aide du code MARS (LAZURE \& DUMAS, 2007) sur l'ensemble $\mathrm{du}$ Golfe de Gascogne (résolution spatiale de $0,005^{\circ}$, sorties toutes les $10 \mathrm{~min}$ ). Les forçages météorologiques (vent et pression) proviennent de la ré-analyse globale CFSR (SAHA et al., 2010) et les différents marégraphes de la côte Aquitaine ont permis de valider les niveaux d'eau calculés. 


\section{XII ${ }^{\text {èmes }}$ Journées Nationales Génie Côtier - Génie Civil \\ Cherbourg, 12-14 juin 2012}

A partir de ces simulations numériques, sont extraites les données utilisées pour l'analyse statistique en probabilités conjointes pour la baie de Saint-Jean-de-Luz. Nous disposons ainsi de 24 années communes entre les données de vagues et de niveaux. La position géographique des points d'extraction est montrée en figure 1a) (sur l'isobathe $50 \mathrm{~m}$ pour les vagues et proche de l'isobathe $20 \mathrm{~m}$ pour les niveaux d'eau).

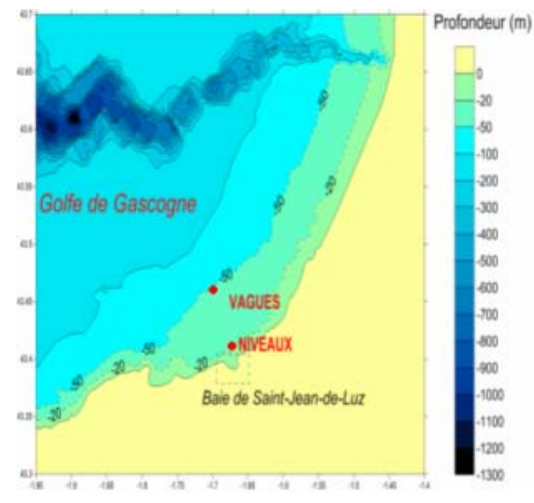

a)

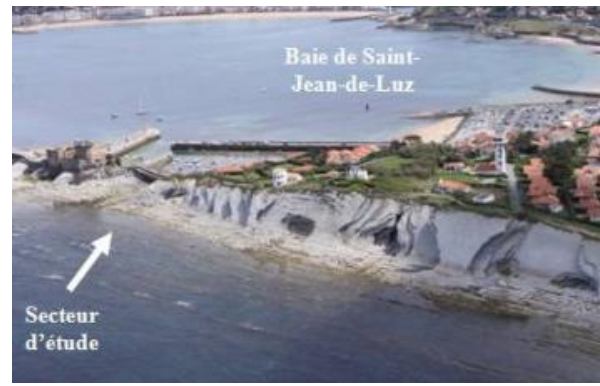

b)

Figure 1. a) Localisation du site d'étude et des points d'extraction des séries temporelles de vagues et de niveaux. b) Photographie du site (par M. Le Collen, 18/04/10). La flèche indique le point de comparaison des deux méthodes du §3.2.

Par la suite, tous les niveaux d'eau présentés concernant le site d'étude sont définis par rapport au zéro hydrographique.

\section{Résultats}

\subsection{Analyse statistique}

Avant de commencer l'analyse proprement dite, un grand soin doit être apporté à la préparation du jeu de données initial. Afin de s'assurer de l'indépendance des triplets $\left(\mathrm{H}_{\mathrm{s}}, \mathrm{SWL}, \mathrm{T}_{\mathrm{p}}\right)$, pour les sites à marées, un ré-échantillonnage des séries temporelles en ne sélectionnant que les triplets aux conditions de pleine mer est effectué (à l'aide d'un script Matlab), ce qui fournit environ 706 observations par an. Ceci est justifié par le fait que les évènements d'intérêt (submersion, franchissement, rupture d'ouvrage...) se produisent la plupart du temps à marée haute. En pratique, une fois les niveaux d'eau de pleine mer identifiés, les hauteurs significatives de vague associées (et périodes correspondantes) sont recherchées en prenant le maximum de $\mathrm{H}_{\mathrm{s}}$ dans une fenêtre de +/- 3 heures centrée sur chaque pic de pleine mer.

Pour le calcul des distributions marginales, au lieu d'utiliser les distributions par défaut de Join-Sea qui sont basées sur les données communes de vagues et de niveaux (24 ans), nous avons réajusté des distributions GPD en utilisant l'ensemble des données disponibles (45 et 31 années pour les données de vagues et de niveaux respectivement). 
Par ailleurs, pour le calcul des extrêmes de SWL, nous avons utilisé une procédure semblable à BARDET et al. (2011). Il s'agit de distinguer dans le signal de niveau d'eau la part déterministe (marée) de la part non déterministe (surcotes), de calculer la distribution des surcotes de pleine mer puis de convoluer les deux signaux (SIMON, 1994). Les résultats sont présentés figure 2 . Le niveau d'eau centennal est de $5,25 \mathrm{~m}$ ce qui est conforme avec la valeur de SIMON (2008) issue de l'analyse des enregistrements du marégraphe de Socoa.

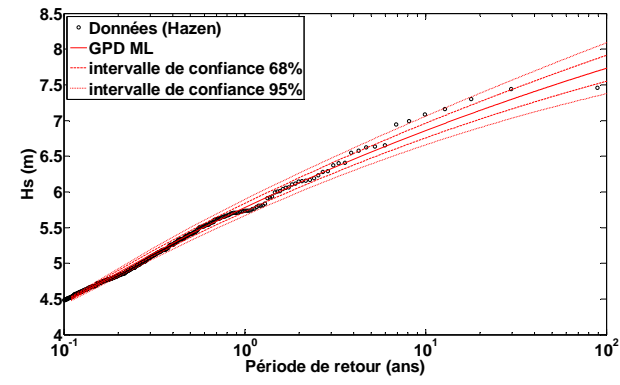

a)

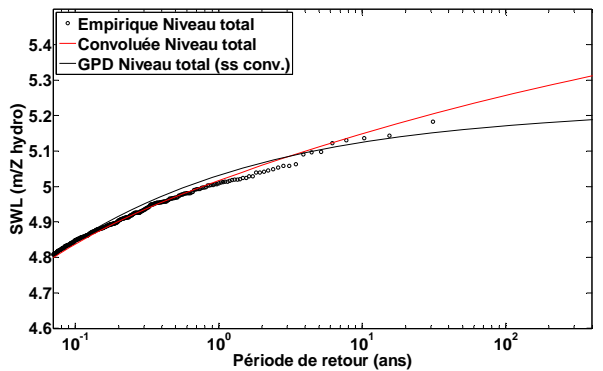

b)

Figure 2. a) Distribution marginale pour $H_{s}$. Paramètres de la loi GPD calculés par la méthode du maximum de vraisemblance. Seuil 4,20 m. b) Distribution marginale pour SWL. En rouge, loi GPD pour les surcotes de pleine mer calculée avec la méthode du maximum de vraisemblance (seuil 0,16 m), convoluée avec la distribution des hauteurs de marée de pleine mer. En noir, loi GPD ajustée directement aux données de SWL (sans analyse en composante harmonique et convolution) avec un seuil à 4,61 m.
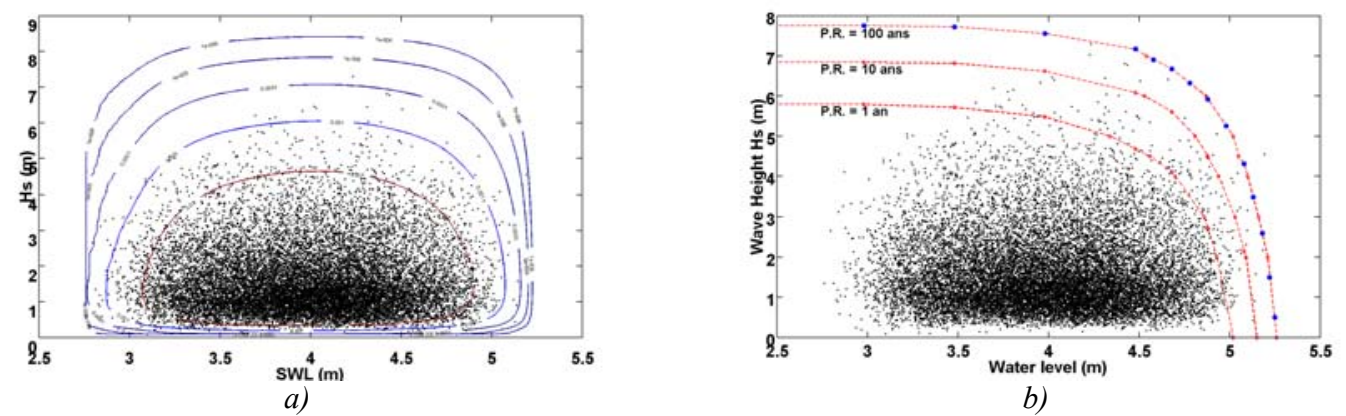

Figure 3. a) Contours d'iso-densité de probabilité conjointe pour $H_{s}$ et SWL. b) Courbes d'iso-période de retour de dépassement conjoint pour $H_{s}$ et $S W L$ calculées sur une période simulée de 10000 ans. Les points noirs représentent les données initiales.

Le modèle de dépendance entre $\mathrm{H}_{\mathrm{s}}$ et SWL est représenté par une loi de distribution normale centrée bi-variée (BVN) dans l'espace gaussien, ajustée au-dessus d'un seuil (les $20 \%$ des valeurs les plus élevées), avec un coefficient de corrélation $\rho=0,035$. La figure 3a) montre des contours d'iso-densité de probabilité conjointe superposés aux données initiales (points noirs) dans l'espace réel. Les courbes d'iso-période de retour 


\section{XII ${ }^{\text {èmes }}$ Journées Nationales Génie Côtier - Génie Civil \\ Cherbourg, 12-14 juin 2012}

de dépassement conjoint pour $\mathrm{H}_{\mathrm{s}}$ et SWL calculées à partir d'une simulation de Monte Carlo de 10000 ans sont représentées figure 3b) pour les périodes de retour 1 an, 10 ans et 100 ans. La distribution conditionnelle des cambrures de vague connaissant $\mathrm{H}_{\mathrm{s}}$ est modélisée dans l'espace gaussien par une régression normale sur $\mathrm{H}_{\mathrm{s}}$ lorsque les hauteurs significatives dépassent $4,05 \mathrm{~m}$.

\subsection{Comparaison des approches pour calculer la période de retour d'un niveau marin}

Les deux approches présentées au $\$ 2.2$ ont été comparées sur le site de Saint-Jean-deLuz. Le point où est calculé le niveau marin total à la côte est situé à l'Ouest de la baie de Saint-Jean-de-Luz, à proximité du Fort de Socoa sur la commune de Ciboure (figure 1b)). Les "évènements" utilisés dans la première approche ont été créés en discrétisant la courbe centennale de dépassement conjoint en 14 points (points bleus sur la figure $3 b)$ ) et en associant une période pic à chacun de ces points (médiane des $T_{p}$ simulées correspondant à un $\mathrm{H}_{\mathrm{s}}$ donné). Les directions pics ont toutes été prises égales à $315^{\circ}$ (i.e. direction Nord-Ouest; choix justifié par la distribution des directions). Quatorze "évènements" ont donc été propagés à la côte à l'aide du modèle SWAN. Le niveau marin extrême centennal est obtenu pour le scénario 11 et atteint $5,37 \mathrm{~m}$ (tableau 1).

Tableau 1. Valeurs du niveau marin statique obtenu au point d'étude après propagation avec SWAN des 14 points sur la courbe centennale de dépassement conjoint. La direction pic des vagues est $315^{\circ}$.

\begin{tabular}{lcccccccccccccc}
\hline Scénario & 1 & 2 & 3 & 4 & 5 & 6 & 7 & 8 & 9 & 10 & $\mathbf{1 1}$ & 12 & 13 & 14 \\
\hline $\boldsymbol{H}_{\boldsymbol{s}}(\boldsymbol{m})$ & 7,76 & 7,73 & 7,56 & 7,17 & 6,91 & 6,68 & 6,32 & 5,92 & 5,26 & 4,31 & $\mathbf{3 , 5 0}$ & 2,60 & 1,50 & 0,50 \\
$\boldsymbol{S W L}(\boldsymbol{m})$ & 2,98 & 3,48 & 3,98 & 4,48 & 4,58 & 4,68 & 4,78 & 4,88 & 4,98 & 5,08 & $\mathbf{5 , 1 3}$ & 5,18 & 5,22 & 5,25 \\
$\boldsymbol{T}_{\boldsymbol{p}}(\boldsymbol{s})$ & 16,5 & 16,4 & 16,3 & 16,2 & 16,1 & 16 & 15,9 & 15,6 & 15,2 & 14,4 & $\mathbf{1 4 , 2}$ & 13,4 & 11,7 & 10,1 \\
\hline $\boldsymbol{S e t u p}(\boldsymbol{m})$ & 0,70 & 0,75 & 0,57 & 0,49 & 0,51 & 0,45 & 0,46 & 0,38 & 0,36 & 0,28 & $\mathbf{0 , 2 4}$ & 0,15 & 0,05 & 0,01 \\
\hline $\boldsymbol{N t o t}(\boldsymbol{m})$ & 3,68 & 4,23 & 4,55 & 4,97 & 5,09 & 5,13 & 5,24 & 5,26 & 5,34 & 5,36 & $\mathbf{5 , 3 7}$ & 5,33 & 5,27 & 5,26 \\
\hline
\end{tabular}

Le calcul du setup a également été effectué directement à partir des 14 conditions de forçages, sans propagation, à l'aide de la formule empirique de STOCKDON et al. (2006) :

$$
\text { setup }=0,35 \beta \sqrt{H_{s} L_{o}}
$$

Avec $\beta$, la pente de la plage, $L_{o}$, la longueur d'onde des vagues (reliée à la période). La pente a été prise égale à $2 \%$ après étude de la bathymétrie locale du site. Le niveau marin extrême centennal est obtenu pour le scénario 12 et atteint $5,36 \mathrm{~m}$. Dans le cas présent, la méthode de calcul du setup (à partir d'une modélisation numérique ou par une formule empirique) influe donc peu sur le résultat final. Cependant, en général, on préfèrera utiliser un modèle numérique pour propager les scénarii et calculer le setup car 
cela permet de prendre en compte les processus physiques de propagation et les spécificités locales du site (bathymétrie, anfractuosités de la côte...).

Pour la deuxième approche, la formule de setup de STOCKDON et al. (2006) a été utilisée pour calculer le niveau marin statique à la côte (voir Eq (1) sans le runup) pour chaque triplet $\left(\mathrm{H}_{\mathrm{s}}, \mathrm{SWL}, \mathrm{T}_{\mathrm{p}}\right)$ simulé par Join-Sea. Les extrêmes de niveau marin ont ensuite été calculés suivant la méthode Join-Sea, i.e. par "counting back" : pour une simulation de 10000 ans, le niveau marin centennal est la $100^{\text {ème }}$ valeur la plus élevée en partant du maximum. Les résultats sont présentés figure 4.

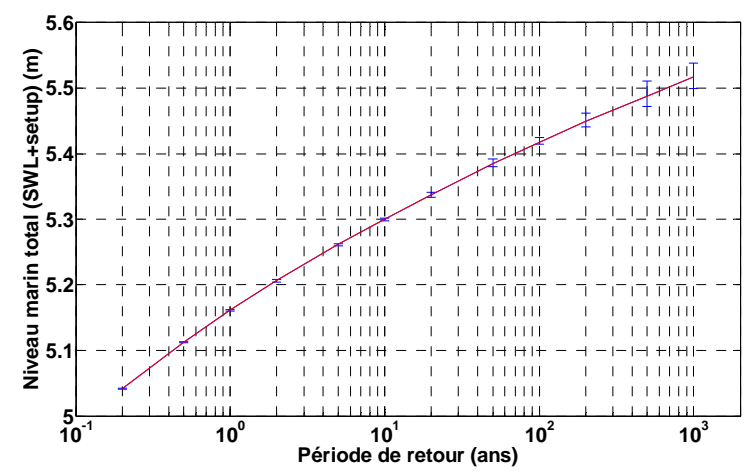

Figure 4. Valeurs extrêmes du niveau marin à la côte en utilisant la formule du setup de Stockdon avec une pente de 2\%. Les bornes des barres d'erreur correspondent aux min et max des valeurs extrêmes obtenues sur 10 simulations de 10000 ans.

La comparaison des deux approches pour la période de retour 100 ans montre que l'approche "variable de structure" est plus sécuritaire $(5,42 \mathrm{~m})$ que l'approche "propagation de scénarii" $(5,37 \mathrm{~m})$. Cette dernière a néanmoins l'avantage d'utiliser un modèle numérique pour la propagation des scénarii prenant en compte les spécificités locales du site étudié et pouvant fournir un résultat en $2 \mathrm{D}$ sur tout un linéaire côtier. Le niveau marin centennal obtenu est supérieur à la valeur que l'on obtiendrait en ne considérant pas les vagues (i.e. 5,25 m, cf. §3.1). Toutefois, l'ajout de l'effet des vagues s'accompagne d'une certaine incertitude. En effet, l'hypothèse fondamentale de la méthode "propagation de scénarii" est l'assimilation de la période de retour du niveau marin à la côte à celle du couple de forçages au large menant à ce niveau marin parmi les 14 scénarii choisis. Or, d'autres combinaisons de forçages (de période de retour conjointe différente de 100 ans) peuvent mener au même niveau marin à la côte. La non-prise en compte de ces combinaisons sous-estime l'effet des vagues et donc le niveau marin centennal (HAWKES et al., 2002). De plus, en se basant sur les couples $\left(\mathrm{H}_{\mathrm{s}}, \mathrm{SWL}\right)$ de la courbe de dépassement conjoint, on perd l'information sur la période pic des vagues qui peut varier beaucoup pour une hauteur de vague donnée.

La seconde approche permet de s'affranchir des principaux inconvénients de la première en utilisant la densité de probabilité conjointe des variables $\left(\mathrm{H}_{\mathrm{s}}, \mathrm{SWL}, \mathrm{T}_{\mathrm{p}}\right)$ 


\section{XII ${ }^{\text {èmes }}$ Journées Nationales Génie Côtier - Génie Civil \\ Cherbourg, 12-14 juin 2012}

calculée par Join-Sea. Un avantage supplémentaire est que l'on a accès rapidement à l'intégralité de la distribution de probabilité de la variable de structure. L'utilisation d'une formule empirique n'est cependant pas adaptée à tous les sites car elle nécessite certaines hypothèses et conditions (e.g. type de plage, type de sédiment, absence d'ouvrages, etc). La formule du setup de STOCKDON et al. (2006) par exemple, n'est valable que pour des plages non anthropisées, larges et très exposées au vagues, et utilise une valeur constante pour la pente de la plage. De plus, les résultats peuvent varier significativement en fonction de la formule empirique choisie.

Les valeurs obtenues par les deux méthodes pour le niveau marin centennal à la côte sont supérieures au niveau extrême de référence du SHOM au port de Socoa (voir tableau 2). Ceci s'explique notamment par la prise en compte du setup dans le calcul du niveau total. Toutefois, les résultats sont à manipuler avec précaution car outre les sources d'incertitudes des deux méthodes présentées plus haut, les données initiales sont issues de modèles (WaveWatchIII et MARS) qui présentent des limites, notamment sur la représentation des extrêmes.

Tableau 2. Niveaux centennaux à la côte. Port de Socoa pour le SHOM (source: SIMON, 2008), point d'étude de la figure 1b) pour le BRGM.

\begin{tabular}{llcc}
\hline Source & Phénomènes & Niveau $\mathbf{1 0 0}$ ans / IGN69 (m) & Niveau 100 ans / Z Hydro (m) \\
\hline SHOM & Marée + Surcote & 3,08 & 5,25 \\
BRGM & $\begin{array}{l}\text { Marée + Surcote (cf. figure 2b)) } \\
\text { Marée + Surcote + Setup : }\end{array}$ & 3,08 & 5,25 \\
& $\begin{array}{l}\text { approche "propagation de scenarii" } \\
\text { Marée + Surcote + Setup : } \\
\text { approche "variable de structure" }\end{array}$ & 3,20 & 5,37 \\
\hline
\end{tabular}

\section{Conclusion}

L'utilisation des probabilités conjointes permet de mieux caractériser les conditions hydrodynamiques d'un site et représente un avantage certain pour améliorer le calcul des périodes de retour de niveaux marins à la côte. La méthode d'analyse statistique Join-Sea est robuste et bien adaptée à cette application. Elle permet en outre de mieux comprendre les processus physiques à l'origine d'un niveau d'eau exceptionnel. La comparaison de deux approches permettant de calculer la période de retour d'un niveau marin à la côte à partir de l'analyse statistique effectuée sur des données acquises au large, a montré que le niveau marin centennal est plus élevé dans le cas de l'approche "variable de structure" $(5,42 \mathrm{~m})$ que dans le cas de l'approche "propagation de scenarii" $(5,37 \mathrm{~m})$, les deux valeurs étant supérieures au niveau de référence du SHOM $(5,25 \mathrm{~m})$. Le choix de l'une ou l'autre de ces deux méthodes complémentaires dépendra des données à disposition et de la nature du site. Les résultats de cette étude sont à 
manipuler avec précaution du fait des incertitudes et approximations intrinsèques à la méthodologie présentée et aux jeux de données disponibles.

Remerciements : La base de données de vagues BoBWA a été constituée dans le cadre d'un projet de recherche associant le BRGM et CNRM-GAME (Météo-France - CNRS) au travers de la thèse d'E. Charles, bénéficiaire d'une bourse AXA de doctorat du Fonds AXA pour la recherche. Sont également remerciés F. Ardhuin (IFREMER) et l'équipe ERA-40. Les auteurs remercient D. Idier pour ses remarques ainsi que P. Hawkes et l'IFREMER pour avoir fourni les codes Join-Sea et MARS respectivement.

\section{Références bibliographiques}

BARDET L., DULUC, C.-M., REBOUR V., L'HER J. (2011). Regional frequency analysis of extreme storm surges along the French coast. Natural Hazards and Earth System Sciences, 11, pp 1627-1639. doi:10.5194/nhess-11-1627-2011

CHARLES E., IDIER D., THIEBOT J., LE COZANNET G., PEDREROS R., ARDHUIN F., PLANTON S. (sous presse). Wave climate variability and trends in the Bay of Biscay from 1958 to 2001. Journal of Climate. doi:10.1175/JCLI-D-11-00086.1

DEFRA (2005). Joint Probability: Dependence Mapping and Best Practice: Technical report on dependence mapping. R\&D Technical Report FD 2308/TR1. Flood and Coastal Defence R\&D Programme.

HAWKES P.J., GOULDBY B.P., TAWN J.A., OWEN M.W. (2002). The joint probability of waves and water levels in coastal engineering design. Journal of Hydraulic Research, Vol. 40, n 3, pp 241-251. doi:10.1080/00221680209499940

HR WALLINGFORD, LANCASTER UNIVERSITY (2000). The Joint Probability of Waves and Water Levels: JOIN-SEA - A rigorous but practical new approach. Report SR537.

LAZURE P., DUMAS F. (2007). An external-internal mode coupling for a 3D hydrodynamical model for applications at regional scale (MARS). Advances in Water Resources. doi:10.1016/j.advwatres.2007.06.010

SAHA, S. and Coauthors. (2010). The NCEP Climate Forecast System Reanalysis. Bulletin of the American Meteorological Society, 91, 1015.1057. doi: 10.1175/2010BAMS3001.1

SIMON B. (1994). Les niveaux marins extrêmes le long des côtes de France et leur évolution. Rapport SHOM 001/94.

SIMON B. (2008). Statistiques des niveaux marins extrêmes de pleine mer Manche et Atlantique. CD-ROM. ISBN : 978-2-11-098357-2, référence CETMEF : C 08-05.

STOCKDON H.F., HOLMAN R.A., HOWD P.A., SALLENGER Jr A.H. (2006). Empirical parameterization of setup, swash and runup. Coastal Engineering 53, pp 573-588. doi:10.1016/j.coastaleng.2005.12.005 